Preprint to be cited as: Debnath, R., Darby, S., Bardhan, R., Mohaddes, K., \& Sunikka-Blank, M. (2020, March 27). A nested computational social science approach for deep-narrative analysis in energy

policy research. SocArXiv. March 27. https://doi.org/10.31235/osf.io/hvcb5

\title{
A nested computational social science approach for deep- narrative analysis in energy policy research
}

\author{
Ramit Debnath*a,b, Sarah Darbyc, Ronita Bardhan ${ }^{\mathrm{a}}$, Kamiar Mohaddes ${ }^{\mathrm{b}, \mathrm{d}}$, Minna Sunikka- \\ Blank $^{\mathrm{a}}$ \\ aBehaviour and Building Performance Group, The Martin Centre for Architectural and Urban Studies, Department of \\ Architecture, University of Cambridge, Cambridge CB2 1PX, United Kingdom \\ bEnergy Policy Research Group, Judge Business School, University of Cambridge, Cambridge CB2 1AG, United Kingdom \\ 'Energy Program, Environmental Change Institute, University of Oxford, Oxford OX1 3QY, United Kingdom
}

dJudge Business School, University of Cambridge, CB2 1AG, United Kingdom

\section{Abstract}

Text-based data sources like narratives and stories have become increasingly popular as critical insight generator in energy research and social science. However, their implications in policy application usually remain superficial and fail to fully exploit state-of-the-art resources which digital era holds for text analysis. This paper illustrates the potential of deep-narrative analysis in energy policy research using text analysis tools from the cutting-edge domain of computational social sciences, notably topic modelling. We argue that a nested application of topic modelling and grounded theory in narrative analysis promises advances in areas where manual-coding driven narrative analysis has traditionally struggled with directionality biases, scaling, systematisation and repeatability. The nested application of the topic model and the grounded theory goes beyond the frequentist approach of narrative analysis and introduces insight generation capabilities based on the probability distribution of words and topics in a text corpus. In this manner, our proposed methodology deconstructs the corpus and enables the analyst to answer research questions based on the foundational element of the text data structure. We verify the theoretical and epistemological fit of the proposed nested methodology through a meta-analysis of a state-of-the-art bibliographic database on energy policy, narratives and computational social science. We find that the nested application contributes to the literature gap on the need for multidisciplinary methodologies that can systematically include qualitative evidence into policymaking.

Keywords: energy policy; narratives; topic modelling; computational social science; text analysis; methodological framework

\section{*corresponding author}

Behaviour and Building Performance Group, The Martin Centre for Architectural and Urban Studies, Department of Architecture, University of Cambridge, Cambridge CB2 1PX, United Kingdom. Email: rd545@cam.ac.uk 
Preprint to be cited as: Debnath, R., Darby, S., Bardhan, R., Mohaddes, K., \& Sunikka-Blank, M. (2020, March 27). A nested computational social science approach for deep-narrative analysis in energy policy research. SocArXiv. March 27. https://doi.org/10.31235/osf.io/hvcb5

\section{Introduction}

The energy policymaking needs in today's world cannot be met alone by the existing methods of quantification due to the multi-agent nature of energy justice and its associated challenges (Ozawa, Chaplin, Pollitt, Reiner, \& Warde, 2019). Clearer representation of these deep interdependencies is well-captured by the United Nation's 17 Sustainable Development Goals (SDGs) that provides a bird's eye view of the global policy and technology challenges. An argument often asserted by policymakers and scientists is that, in spite of 'sound' technology and 'rational' policies, policy implementation yields unexpected results. It is often due to the lack of multidisciplinary insights into the energy policymaking and implementation processes (Ozawa et al., 2019).

The current regime of energy policymaking by scientists and technologists is guided by the promotion of preferred technologies with theoretical properties that complements the objectives of the leading political forces. They justify their proposition based on quantitative models of energy and climate systems that graft directional biases in the policymaking often overlooking the strengths of qualitative stakeholder deliberations. 'Good' energy policy under a technocratic directional bias thus becomes policy focussed on 'getting the technology right' (Ozawa et al., 2019). While technology can indeed be force to achieve climate change mitigation and sustainability goals, its intended welfare effects may be restricted if the wants of a society are not appropriately reflected in policy (MacKay, 2008). Non-economic drivers may be crucial in achieving the welfare effects from specific energy and climate policy, they remain underrepresented in the current literature. So much so that, even the work of the Intergovernmental Panel on Climate Change(IPCC) has been characterised as highly directional and 'unidisciplinary' as it is based on a clear separation between the natural sciences and social sciences, and a understanding that social sciences are based on natural sciences (Vasileiadou, Heimeriks, \& Petersen, 2011). The problem with such technocratic directionality bias is that it leads to an inherent limitation in the definition of policy goals, which become defined as 'reasonable' or 'rational' according to technical parameters.

Purely technical approaches cannot account for the multidimensional nature of the sociocultural forces that effects policy implementation. Oversimplification of policies through quantitative and frequentist generalization of contexts remain energy policymaking challenges. Therefore, Ozawa et al., (2019) state that the premise of 'good' energy policy is much more multi-layered, nuanced and non-obvious. Directional bias among policymakers and scientists misses this premise. A multidisciplinary perspective to modern-day energy policymaking requires a deep understanding of historical and of social, political and cultural institutions that constitute the people, places and practices. It demands serious inclusion of social science and humanities thinking in energy policy, thereby leading to a much richer set of insights for 'good' energy policy in the $21^{\text {st }}$ Century. However, an inherent problem with the qualitative approaches of social science and humanities for policy applications remains with the directionality of its application. It can cause inferential bias that leads to issues of reliability, validity, replicability and generalisability in qualitative research (Trotter, 2012).

A critical and relatively new object of energy and climate change research through the lens of social science and humanities is the use of narratives as a way of crystallising arguments and assumptions (Mithra Moezzi, Janda, \& Rotmann, 2017b). So much so that narratives, stories and storytelling have garnered credible representation in energy and climate change research and policy (Janda \& Topouzi, 2015; Lertzman, 2015; M. Moezzi, 2015; Randall, 2009; Rotmann, Goodchild, \& Mourik, 2015). However, the value of these narratives depends significantly on their interpretation by 
Preprint to be cited as: Debnath, R., Darby, S., Bardhan, R., Mohaddes, K., \& Sunikka-Blank, M. (2020, March 27). A nested computational social science approach for deep-narrative analysis in energy policy research. SocArXiv. March 27. https://doi.org/10.31235/osf.io/hvcb5

researchers and practitioners (Mithra Moezzi et al., 2017b). This will induce biases in the interpretability of the results. Some authors claim that this makes them ineffectual as compared to science and technology-driven quantitative methods (Bazeley, 2004; Fukuyama \& Weber, 2009); however, it is worth bearing in mind that methods based on the 'hard' sciences will also have inbuilt biases, for example in the choice of metrics or indicators. They are also likely to miss nuances in the use of language and thus to lose some of the meaning intended by narrators. On the contrary, a human who is fully conversant with the language used can capture the contextual socio-cultural gradations by using robust qualitative methodological tools like grounded theory.

The primary contribution of this paper is to improve the robustness of narrative analysis in public policy research. Here, we focus exclusively on energy policy applications. In doing so, we propose a nested methodological framework based on current state-of-the-art techniques of data collection, qualitative analysis and computational social sciences. The novel nested approach presented here contributes to lessening directional biases in narrative-driven policy analysis. The innovation of the study lies in the nested treatment of two epistemologically parallel but methodologically distinct concepts (Baumer, Mimno, Guha, Quan, \& Gay, 2017) of topic modelling (TM) and grounded theory (GT). These parallel methods are used to complement each other, to reduce directional bias in energy policy applications.

The aim of this paper is to improve the robustness of narrative-driven results in energy policymaking. By improving the robustness, we want to expand the applicability of text-based tools like narratives, stories and storytelling into the disciplines of evidence-based policymaking (Pawson, 2002). While the experience of a qualitative researcher remains the most critical control variable for narrative-driven analysis, manual coding of texts is highly taxing and is limited by human cognitive abilities. At best, it allows for sensitive and insightful analysis, there is always the likelihood of some directional and researchers' bias in the complex narrative analysis (Bryant \& Charmaz, 2019). We enrich the manual-coding processes of narrative analysis by employing artificial intelligence (AI) and machine-learning aided topic detection algorithm, i.e., topic modelling (TM), that can, if welldesigned, lessen directionality biases. Recent studies have shown that qualitative researchers, guided by the principles of grounded theory (Glaser \& Strauss, 1967; Strauss \& Corbin, 1997) have incorporated a combination of manual-coding methodologies like open coding, axial coding and selective coding to improve the robustness of their analysis (Bryant \& Charmaz, 2019; Williams \& Moser, 2019). Our proposed methodology replaces these steps with a probabilistic step of 'word' selection using TM that statistically improves the robustness of results. This step lessens directionality bias as TM deconstructs the text-corpus of the narratives into its fundamental constituents (i.e., words) and aids the analyst to reconstruct the narratives through a GT driven-interpretivist approach.

The proposed deep narrative analysis framework presented here does not intend to replace each of these distinct methods. Instead, it shows the synergistic capability of such methods that contributes to the growing literature of computational social sciences and energy policy research. The central function of the proposed nested methodology is to deconstruct a text corpus collected as narratives and identify patterns among word-word, word-topic and topic-topic probabilities in the corpus. It is intended to remove directionality biases in order to enable an analyst to derive clearer insights from the narratives through grounded theory for policy applications. We establish this claim through a science mapping-based meta-analysis that verifies its theoretical fit.

Deconstruction of the raw-narratives into their fundamental elements (words), and then reconstructing them through an interpretivist GT application not only removes directionality biases, 
Preprint to be cited as: Debnath, R., Darby, S., Bardhan, R., Mohaddes, K., \& Sunikka-Blank, M. (2020, March 27). A nested computational social science approach for deep-narrative analysis in energy policy research. SocArXiv. March 27. https://doi.org/10.31235/osf.io/hvcb5

but it also preserves the cognitive essence of a narrative-analysis. To the best of our knowledge, we believe such a nested approach has never been developed or applied to humanities and social science bounded evidence-based energy policy research. Our proposed framework contributes to the growing field of computational social sciences while expanding the applicability of rigorous qualitative methods like GT-based narrative analysis. While in this paper we develop our proposed nested approach, in Debnath et. al. (2020) we illustrate the application of the proposed methodology for deriving energy justice pathways in the Global South.

This paper is structured as follows; section 2 presents a background on the state-of-the-art methods in computational social sciences for text analysis and qualitative methodologies in humanities and social sciences for energy and climate policy applications. Section 3 outlines the proposed deep-narrative analysis framework. Section 4 presents the methodological steps of the bibliometric network analysis employed here to establish the epistemological and meta-theoretical fit of the proposed methodology. The discuss results in section 5 and provide concluding remarks and establish the way forward for the application of the proposed framework in section 6 .

\section{Background}

\subsection{Narrative-analysis in energy policy research}

Narratives, stories and storytelling go beyond the analytical convention of energy and climate change research. It helps in approaching the intersection of nature, humanity, and technology in a multidisciplinary manner, using a lens from social sciences, humanities, and practitioners 'perspectives (Janda \& Topouzi, 2015). A special issue of Energy Research \& Social Science journal, titled 'Narratives and Storytelling in Energy and Climate Change Résearch' (Mithra Moezzi, Janda, \& Rotmann, 2017a) have established importance of narrative, stories and storytelling as critical data objects in energy and climate change research. This special issue presented 34 articles that employ stories and storytelling as avenues of gathering new data and understanding, communication, and influencing others. Narrative analysis in the same issue presented novel ways of crystallising arguments and assumptions that generated deeper policy insights (Mithra Moezzi et al., 2017b).

The energy and climate policy research is however young with a few instances of narrative analysis in the published literature. Bushell, Buisson, Workman, \& Colley, (2017) proposed multiple narrative designs to promote action on climate change and development of strategic-narratives to engage multiple stakeholders better. Hermwille, (2016) advanced the role of narratives in the crystallisation and structuring of arguments in socio-technical energy policy discourse by citing examples from Fukushima and energy regimes of Japan, Germany and the United Kingdom. In doing so, the author captures the policy responses from several perspectives. It is one of the rare application of narratives to generate socio-technical policy responses. However, as mentioned in section 1 , such application is limited by the directionality and interpretability of the researcher, that subconsciously induces bias. Hermwille, (2016) in his concluding remark states that there is a need for explorative methodologies that can unveil contemporary mechanisms of people, places and practices. Structured Dialogic Design Processes (SDDP) is another methodology that wishes to address some of the explicit normative biases of narrative-based analysis by using web-based communication platforms, thus leveraging information and communication technologies (ICT) (Laouris et al., 2009). However, SDDP uses observer-dependent data and entirely depends on engaging stakeholders as "expert observers" of the situation in which they are embedded. While it remains a strength of this method, the reliability of this methodology depends on the ability of the expert observers to take action in their situation 
Preprint to be cited as: Debnath, R., Darby, S., Bardhan, R., Mohaddes, K., \& Sunikka-Blank, M. (2020, March 27). A nested computational social science approach for deep-narrative analysis in energy policy research. SocArXiv. March 27. https://doi.org/10.31235/osf.io/hvcb5

(Bausch, 2008). This may lead to directional biases in its generalised interpretation. A methodological advancement to a similar discourse-analysis through topic modelling was proposed by Jacobs \& Tschötschel, (2019) which provides a critical epistemological clue for this study.

Methodologically there has not been much development in the use and treatment of narratives in energy and climate change research. Most developments are in the mode of data collection using narratives. For example, Shaw \& Corner, (2017) develops a narrative workshop methodology to engage the public in climate change policies. Their innovation lies in the data collection and narrative generation regimes that enable the public to validate their values and identity, but its results embed directionality biases based on the interpretation of the researcher. Smith et al., (2017) uses the concept of 'energy utopias' to create experimental stories and generate narratives to refresh public and political conversations about energy and decarbonisation. They found that stories and narrative by themselves do not drive transformation, but they provide confidence in decision-making and public participation.

Similarly, Howarth, (2017) found that narratives and stories can increase experiential engagement on climate change. Stories and narratives on climate change and low-carbon policy can fill in the information deficit in the present techno-economic models. Our observation from these state-of-theart articles is that the narratives and stories provide excellent multi-stakeholder engagement platforms, but they offer piecemeal solutions to the problem. The methods discussed above have their basis in grounded theory (GT), as each aims to derive a situational-theory based on the narrativeanalysis. We use GT as a foundational component of the nested-model to retain the interpretive effectiveness of narrative-analysis in a computational environment.

Our proposed nested model is based on the foundation of a probability distribution that provides a higher degree of freedom to zoom-in, zoom-out and zoom-through the problem ${ }^{1}$. With zooming out, possibilities and assumptions that are forgotten or are taken for granted can be better recognised; zooming in aids better understanding of the granularity that is hidden in the frequentist summaries of central tendencies, like granularities hidden in often-repeated statements. Most importantly, the ability to zoom through in a computational model can aid in critically analysing narratives as to material reality, norms and practices that determine the energy culture. These three functions cumulatively illustrate our claim of a 'deep-narrative' analysis.

To perform the three deep-narrative analysis functions, we set our methodological framework around computational text analysis using unsupervised machine-learning techniques. In the following section, we present the state-of-the-art application of topic modelling in social science and humanities research that serves as the foundational basis for our proposed deep narrative analysis methodology.

\subsection{Topic modelling in humanities and social science research}

Computational text analysis is an emerging critical methodology in the field of social science and humanities that represents a part of the state-of-the-art in computational social sciences. It centres around probabilistic topic modelling that distributes vocabulary over probability distribution. The high probability words in each distribution can be readily interpreted as recognisable themes, and are thus referred to as "topics" (Baumer et al., 2017; Grimmer \& Stewart, 2013; Jockers, 2013). There 
Preprint to be cited as: Debnath, R., Darby, S., Bardhan, R., Mohaddes, K., \& Sunikka-Blank, M. (2020, March 27). A nested computational social science approach for deep-narrative analysis in energy policy research. SocArXiv. March 27. https://doi.org/10.31235/osf.io/hvcb5

are many methods of topic modelling, of which Latent Dirichlet Allocation (LDA) is the most popular in the field.

LDA is an advanced textual analysis technique grounded in computational linguistics research that calculates the statistical correlations among words in a large set of documents to identify and quantify the underlying (latent) topics in these documents. In linguistic science, Vulić, De Smet, \& Moens, (2011) have used LDA to identify the translation of words among languages. Bauer, Noulas, Seaghdha, Clark, \& Mascolo, (2012) have used topic modelling to analyse millions of textual comments of geographical and temporal check-ins to understand behavioural pattern at a global scale. Lui, Lau, \& Baldwin, (2014) have used a variant of LDA to detect languages in multilingual documents.

Similarly, topic modelling has garnered significant importance in political science and rhetoric analysis (Grimmer \& Stewart, 2013). Balasubramanyan, Cohen, Pierce, \& Redlawsk, (2012) have used an LDA-based topic modelling approach to investigate reactions of different political communities to the same news. Song, Kim, \& Jeong, (2014) have used topic modelling on twitter dataset to analyse the socio-political landscape of the 2012 Korean Presidential Election. In Chen, Zhu, Kifer, \& Lee, (2010), the authors have used an LDA model to reduce the size of auto-discovered latent opinions words and topics in political standpoints between Republicans and Democrats in the US. German National Elections since 1990 was analysed using an extension of LDA (LogicLDA and Labeled LDA) that explored multi-dimensionality of political documents, pushing the limits of content analysis in the social sciences (Zirn \& Stuckenschmidt, 2014). A detailed explanation of topic modelling choices for political content analysis can be found in Grimmer \& Stewart, (2013).

There is a significant body of research ongeographical topic modelling. Yano, Cohen, \& Smith, (2009) have used topic modelling using a geographic LDA (LGTA, Latent Geographical Topic Analysis) to extract geographical information in online political blogs. A multi-scalar LDA model was used by Tang et al., (2013) to cluster very high-resolution panchromatic satellite images. More recently, LDA was used as a means of data-driven geotopic detection of urban emergencies for natural hazards, manmade disasters and other emergencies (Wang \& Taylor, 2019). Topic modelling is now being used to map consumer sentiments and understanding their behavioural choices on a geographical scale for enhancing business potential (Qi, Procter, Zhang, \& Guo, 2019; Toubia, lyengar, Bunnell, \& Lemaire, 2019). Critical environmental and social problems are now being identified using twitter-based geotopics that provide rich knowledge of important events (e.g., cultural activities, political campaigns, accidents, crisis) on an urban scale. Yao \& Wang, (2019) have used a dynamic topic modelling approach to identify urban crisis in US cities that had crucial implications in sustainable city planning and policymaking.

Despite the success of LDA-based topic modelling applications in sociology, history, political science and linguistics, topic models are known to suffer from some conceptual and practical problems, like a lack of justification of Bayesian priors, divergences with statistical characteristics of real texts and the imprecision to choose the relevant number of topics (Gerlach, Peixoto, \& Altmann, 2018). Recent efforts in solving these problems refer to the cross-fertilization between multiple fields through mixed-method or multi-method interdisciplinary approaches (Gerlach et al., 2018). Baumer et al., (2017) compared grounded theoretic approach with topic modelling to compare interpretive social sciences (grounded theory) with statistical machine learning (topic modelling). Their results have shown that two analyses produce similar results with field-specific drawbacks. However, their 
Preprint to be cited as: Debnath, R., Darby, S., Bardhan, R., Mohaddes, K., \& Sunikka-Blank, M. (2020, March 27). A nested computational social science approach for deep-narrative analysis in energy policy research. SocArXiv. March 27. https://doi.org/10.31235/osf.io/hvcb5

comparison suggested that novelty of using these techniques lies in a compelling combination of such methods as a complementary entity, rather than a 'replacement' of one method. In this study, we do not intend to compare all the available topic models (see Grimmer \& Stewart, (2013) for such comparative study), neither contribute to the development of such computational models. Here, we derive humanities and social science-oriented application framework for deep-narrative analysis using topic modelling technique. The application of this model is presented in Debnath et al. (2020) of this study.

There are a handful of studies on the application of topic modelling in energy research. For example, Jiang, Qiang, \& Lin, (2016) applied topic modelling on a bibliometric dataset on hydropower research. They established 29 topics that described the intellectual architecture of hydropower research and found that an interdisciplinary lens in hydropower research is needed for higher-level policy benefits. The authors also established that topic modelling approach in energy research could provide a new outlook to evidence-based policymaking. More recently, Walker, Chandra, Zhang, \& van Witteloostuijn, (2019) have verified the critical importance of topic modelling-based approaches in deriving evidence for policy design and policymaking in public administration.

\section{Conceptual framework of the proposed nested deep-narrative analysis methodology}

The nested deep-narrative analysis methodology proposed in this study is illustrated in Fig 1. The conceptual framework presented in Fig 1 has three core parts that work in synergy with each other. The nested methodological arrangement is to efficiently extract complementary benefits of topic modelling (TM) and grounded theory (GT). The importance of narrative-analysis in energy policy research was discussed in section 2.1 with evidence from state-of-the-art literature. Also, the application spectrum and robustness criteria of topic modelling as a computational social science research tool were presented in section 2.2. We claim that the introduction of topic modelling in the narrative analysis will not only improve the robustness of the findings for policy application, but it will also reduce directionality bias in the research. We refer to directionality bias as the biases that are consciously or sub-consciously induced in the interpretation of narrative-driven research that alter the objective meaning of the findings. These assumptions are coherent with the arguments of Trotter, (2012) and Bryant \& Charmaz, (2019). Moreover, from a pure applied public policy perspective, Walker et al., (2019) have argued the need for objective and robust qualitative approaches in evidence-based policymaking. They also point out that the current qualitative methods have a strong directionality quotient (referred to as 'hegemonic interpretation', pp 475) that is sensitive to the interpretabilitybias of the researcher, analyst or policymaker.

Our proposed methodology aims to address this limitation by improving the objectiveness of narrative analysis though a nested application of unsupervised machine-learning driven TM while preserving the insight generating capability of narrative by using GT (see Fig 1). The nested approach proposed here provides a remarkable methodological polyvalence to topic modelling and the grounded theory. It is further discussed with bibliometric evidence in section 5 while establishing the epistemology-fit and the meta-theoretical fit. While TM represents the topic detection and clustering 
Preprint to be cited as: Debnath, R., Darby, S., Bardhan, R., Mohaddes, K., \& Sunikka-Blank, M. (2020, March 27). A nested computational social science approach for deep-narrative analysis in energy policy research. SocArXiv. March 27. https://doi.org/10.31235/osf.io/hvcb5

algorithms based on the theories of natural language processing and computational linguistics. The Grounded Theory represents a systematic methodology that conceptualises the latent social patterns and relationships for understanding observed social processes within a specific setting (Noble \& Mitchell, 2016). It is a well-established empirical method in qualitative research and is known for its robustness in inductive research. However, its rigour is highly depended on the quality of manual coding of the raw data and the presence of directionality biases in the narrative-collection.

We overcome these limitations by the nested application of TM and GT through a deconstructivist approach of breaking down narrative corpus into its fundamental word constituents. The words are then clustered through probability distribution functions (steps of TM) that are used in an interpretivist manner through the lens of GT. In doing so, TM clusters high probable words from a text corpus of narratives, GT aids the analyst in crystallising arguments and insights that statistically inform evidence-based energy policymaking. However, we make it clear that TM should be constructed with specific objectives in mind, rather than statistical optimisation. GT, on the other hand, aids in zooming through the topics and the narratives and produce insights based on the probability distribution of the topics. The proof-of-concept of this nested framework (see Fig 1) is presented in Paper-Il of this study.

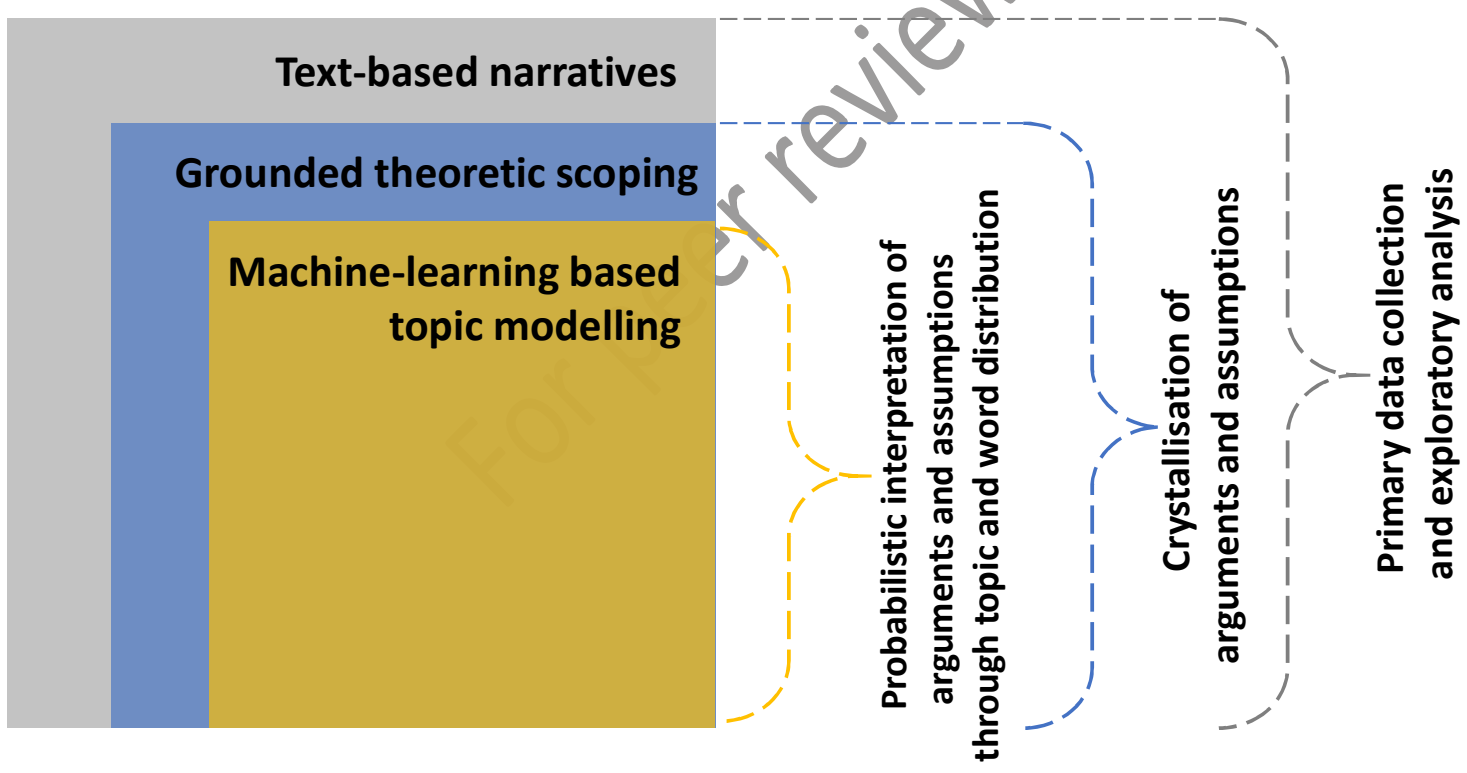

Fig 1. Proposed nested deep-narrative analysis methodological framework based on computational social science and narrative-analysis

\section{Data and method}

A bibliometric network analysis was performed to identify the trends in narrative-based energy policy research and computational social science-based topic modelling through science mapping. A general science mapping workflow was followed as per the guidelines of Börner, Chen, \& Boyack, (2003) that included bibliographic database extraction (Web of Science or Scopus), data analysis using network theory and data visualisation. The results of this network analysis were used to generate evidence from state-of-the-art literature on the epistemological-fit and meta-theoretical fit of nested application of topic modelling, grounded theory and narrative analysis, as per the proposed 
Preprint to be cited as: Debnath, R., Darby, S., Bardhan, R., Mohaddes, K., \& Sunikka-Blank, M. (2020, March 27). A nested computational social science approach for deep-narrative analysis in energy policy research. SocArXiv. March 27. https://doi.org/10.31235/osf.io/hvcb5

conceptual framework. In this study, science mapping is used as a proxy to a meta-analysis of the published work in the state-of-the-art domain of energy policy, narrative analysis, computational social science and topic modelling.

The Web of Science (WoS) bibliometric database was used as the primary data source for the network analysis. A complete bibliometric extraction was conducted that included 'title', 'abstract', 'keywords' and 'journal keywords (keyword plus)'. The WoS database showed 311 published documents between 2000 - 2019 on the topic 'energy policy' and 'narratives' (see Fig 2), referred to as 'keyword set A'. Similarly, the WoS database had revealed 94 papers between $2000-2019$ on the topic 'computational social sciences' and 'topic modelling', referred to as 'keyword set $B$ '. The data analysis was performed using the bibliometrix package in the R-programming language (Aria \& Cuccurullo, 2017).

Data analysis consisted of descriptive analysis and network extraction of the WoS database of 311 articles. Co-word analysis (Callon, Courtial, Turner, \& Bauin, 1983) was used to study the conceptual structure of narrative-driven energy policy research. It produces semantic maps of keywords, titles or even full texts that illustrate the cognitive structure of the interconnected topics within that field (in this study, 'energy policy' and 'narrative'). These maps were further processed using a normalisation function over the relations (edges) between its nodes (vertices) using similarity measures such as Salton's cosine, Jaccard's coefficient, and Pearson's correlation. With the normalised data, various dimensionality reduction techniques such as principal component analysis/factor analysis, multidimensional scaling (MDS) and multiple correspondence analysis (MCA) were to cluster the bibliometric data into appropriate groups. These steps were followed after the best-practice guidelines of Aria \& Cuccurullo, (2017).

Data visualisation was presented through dendrograms, bi-dimensional maps, word-cloud and keyword co-occurrence networks. These maps were created using plotly (Sievert, 2018) and igraph (Csardi \& Nepusz, 2006) packages in R. In additional, network analysis was used to generate statistically treated maps that indicated relationships between word clusters through heliocentric maps (De Moya-Anegón et al., 2005), geometric models (Skupin, 2009) and thematic maps (Cobo, López-Herrera, Herrera-Viedma, \& Herrera, 2011).

Following these analytical procedures, we present a rationale for the compatibility of topic modelling with narrative-analysis through grounded theory using discussions on its meta-theoretical fit and epistemological fit. Additionally, we present the rationale behind the added value of combining topic modelling and narrative-analysis using grounded theory to justify the novelty of the proposed methodological framework in energy policy research. 
Preprint to be cited as: Debnath, R., Darby, S., Bardhan, R., Mohaddes, K., \& Sunikka-Blank, M. (2020, March 27). A nested computational social science approach for deep-narrative analysis in energy policy research. SocArXiv. March 27. https://doi.org/10.31235/osf.io/hvcb5

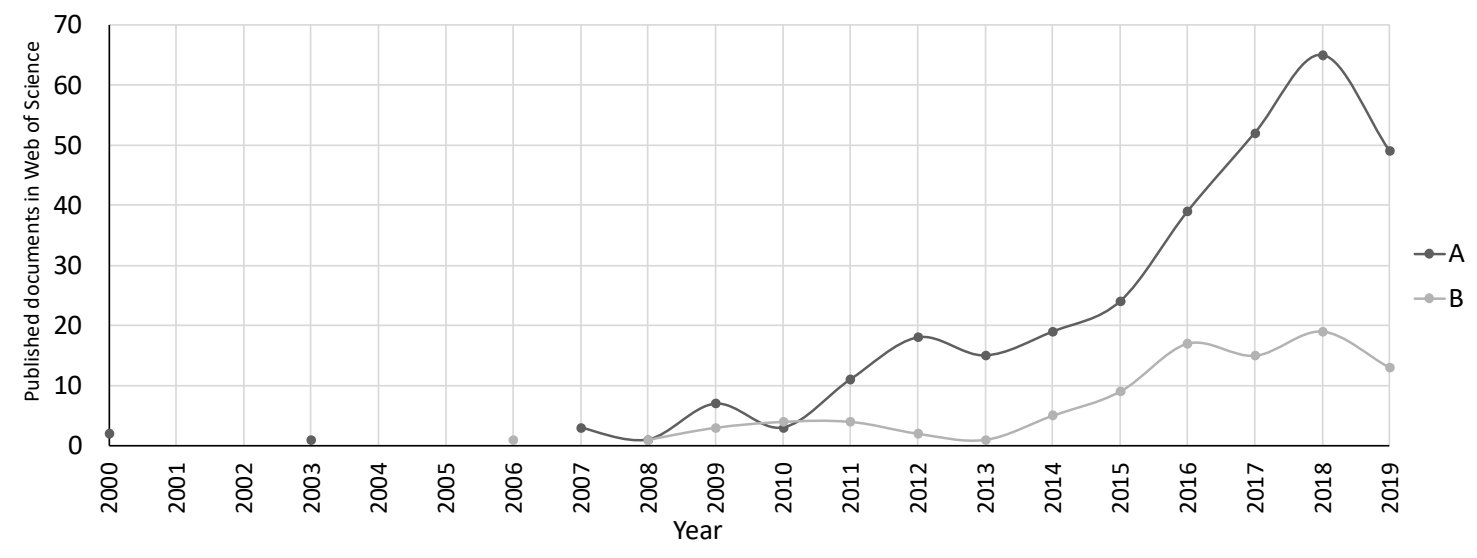

Fig 2. Published documents in Web of Science (WoS) database for the searched keyword set A: 'energy policy' \& 'narratives' ( $n=311)$; and keyword set B: 'computational social science' \& 'topic modelling' $(n=94)$ between 2000 - 2019.

\section{Results and discussion}

The heuristic search of state-of-the-art literature in the Web of Science (WoS) bibliometric database showed 311 documents for the keyword set A ('energy policy' \& 'narrative') and 94 documents for the keyword set B ('computational social science' \& 'topic modelling') as shown in Fig 2. The common words in these keyword set are illustrated in Fig 3 which word-cloud of high-frequency words under keyword set A and keyword set B. Fig 3 shows critical exploratory evidence of the policy applications of topic modelling through public opinion and discourse analysis (see Fig 3b). It further shows that the use of narratives in energy policy has greater use in governance, politics, management, uncertainty analysis and perspective building towards sustainability (see Fig 3a). It provides a strong indication concerning the suitability of topic modelling for energy policy applications. Thus, supporting our initial assumption on the complementary application of topic modelling (TM) with grounded theory (GT) for deep-narrative analysis. 
Preprint to be cited as: Debnath, R., Darby, S., Bardhan, R., Mohaddes, K., \& Sunikka-Blank, M. (2020, March 27). A nested computational social science approach for deep-narrative analysis in energy policy research. SocArXiv. March 27. https://doi.org/10.31235/osf.io/hvcb5

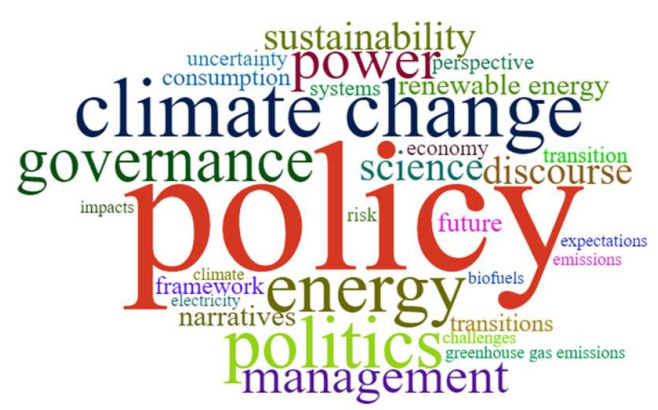

a) Keyword set $A$

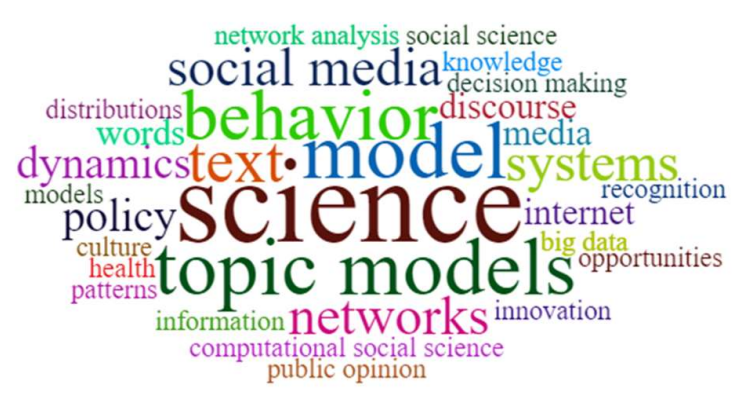

b) Keyword set B

Fig 3. Word-clouds illustrating high frequency words in the WoS database for search keyword set A: energy policy and narrative $(n=311)$, and search keyword set B: computational social science and topic modelling $(n=94)$.

\section{Meta-theoretical fit of the proposed methodology}

As discussed in section 3, most of the narrative analysis in energy policy research is employed to crystallise arguments and assumptions. GT is employed to understand the latent social patterns and relationships of observed social processes within a specific setting bounded by the narratives. It entrails that our nested approach presents narrative-driven insights as an objective entity that arises from the context a word is employed in and that it is not an inherent feature of the word itself. TM corresponds well with this view of objectivity as a topic is a probability distribution over all the words used in the original text corpus. Each word in principle is represented in each topic, and its meaning varies between topics. It is the analyst's task to interpret the meaning of a topic based on how it ranks terms and how it relates to other topics. Our nested model (see Fig 1 ) guides the analyst in interpreting the topics through GT. Thus, improving the robustness of the narrative-analysis for policy applications.

Similarly, the meanings of a word are topic-specific and based on the other words that appear in the topics in which it features prominently. GT can aid the analyst in connecting the hidden dots between the words and the topics, thus, explaining a broader social process or phenomenon. These arguments exemplify TM and GT as a method well-qualified for deep narrative analysis, our hypothesis. TM and GT align well with the insight generation and argument crystallisation capability of narrative-analysis in policy research, as

- TM explicitly models 'polysemy', i.e., the coexistence of much possible meaning for a word or phrase (DiMaggio, Nag, \& Blei, 2013). It traces the multiplicity of contexts of every word in the text corpus. GT is inductive, and it aids in linking the latent connections between socio-cultural processes that are sensitive to policy variables (in energy research and social science context) (Bryant \& Charmaz, 2019). It implies that TM induces the quality of directness in the interpretation, which adds robustness to the synthesis of the latent social process through GT. Thus, crystallising arguments with probabilistic evidence, we argue it as the 'deep narrative analysis' in energy policy studies.

- Topics themselves obtain their meaning through i) the inter-relationship between words in a cluster of topics; ii) inter-cluster meaning of words between the topics, and iii) through frequent co-occurrences with other topics. Further meaning is derived by linking words to multiple topics, indicating the relationality feature of TM (Jacobs \& Tschötschel, 2019). Additionally, GT strengthens the relationality to develop critical insight for policy applications (Bryant \& Charmaz, 2019). 
Preprint to be cited as: Debnath, R., Darby, S., Bardhan, R., Mohaddes, K., \& Sunikka-Blank, M. (2020, March 27). A nested computational social science approach for deep-narrative analysis in energy policy research. SocArXiv. March 27. https://doi.org/10.31235/osf.io/hvcb5

Jacobs \& Tschötschel, (2019) argues that 'topics could be interpreted as frames, themes, et cetera, but stressed that the most appropriate interpretation depends on how the method is used by the analyst'. We expand on this argument using factor analysis (see Fig 4) on the WoS database of keyword set $A$ and keyword set $B$ to comment on the commonality and cross-fertilisation between $A$ and $B$. We argue that the possibility of this cross-fertilisation provides TM-aided deep-narrative analysis with a notable methodological polyvalence for energy policy research and social sciences. Cross-fertilisation of themes/words from Fig 4a and Fig 4b respectively, shows that the bibliographical landscape concerning TM, narrative-analysis and (energy) policy applications have a commonality in words like 'public opinion'; 'policy'; 'decision-making'; 'discourse'; 'practice'; 'dynamics'. It establishes the meta-theoretical fit of TM with narrative-analysis for policy applications as TM offers a dynamic route to narrative-driven policy research through public opinion synthesis.

However, we maintain a caution that cross-fertilisation of qualitative methods with TM should be constructed with specific research objective in mind, rather than with statistical optimisation. For example, in our proposed nested methodology (see Fig 1), we use TM with GT to perform deepnarrative analysis such that GT aids in the refining the parameters of the model interpretation to facilitate the best possible answer to those research questions. It would mean building the topic models in a way that answers the research questions, rather than adapting research questions so that they can be answered through TM. The later remains a dimitation in the current paradigm of computational social science research for public policy applications (Grimmer \& Stewart, 2013; Jacobs \& Tschötschel, 2019; Roberts, Stewart, \& Tingley, 2016).

Additionally, with the proposed nested approach of TM and GT (see Fig 1), we contribute to the present controversy over how to select the 'right' number of topics (Arun, Suresh, Veni Madhavan, \& Murthy Narasimha, 2010; Griffiths \& Steyvers, 2004; Grimmer \& Stewart, 2013). While TM provides with a perplexity score of the topic as a measure of model convergence (Griffiths \& Steyvers, 2004), GT enables the analyst to qualitatively interpret the topics in the most subjectively conscious manner. We propose one way of doing it is to explore the bibliographic databases (i.e. science mapping) to identify topic clusters based on the state-of-the-art literature on that topic. We apply it in the Part-II of this study.

Similar to our claim on choosing the 'right' number of topics, Jacobs \& Tschötschel, (2019) argues on focussing on one well-delineated meta subject (such as trade policy or the national economy), and by using a corpus that only features a single genre of texts (newspaper articles, speeches, journalistic piece). It is based on the applied research of Törnberg \& Törnberg, (2016b, 2016a) on TM and discourse analysis for social policy. It is to be noted that TM do not automatically conduct narrative analysis with the increase in number of topics, higher number of topics do not by definition mean crystallisation of arguments and assumptions in policy analysis. It aids the analyst in an interpretivist manner. 
Preprint to be cited as: Debnath, R., Darby, S., Bardhan, R., Mohaddes, K., \& Sunikka-Blank, M. (2020, March 27). A nested computational social science approach for deep-narrative analysis in energy policy research. SocArXiv. March 27. https://doi.org/10.31235/osf.io/hvcb5

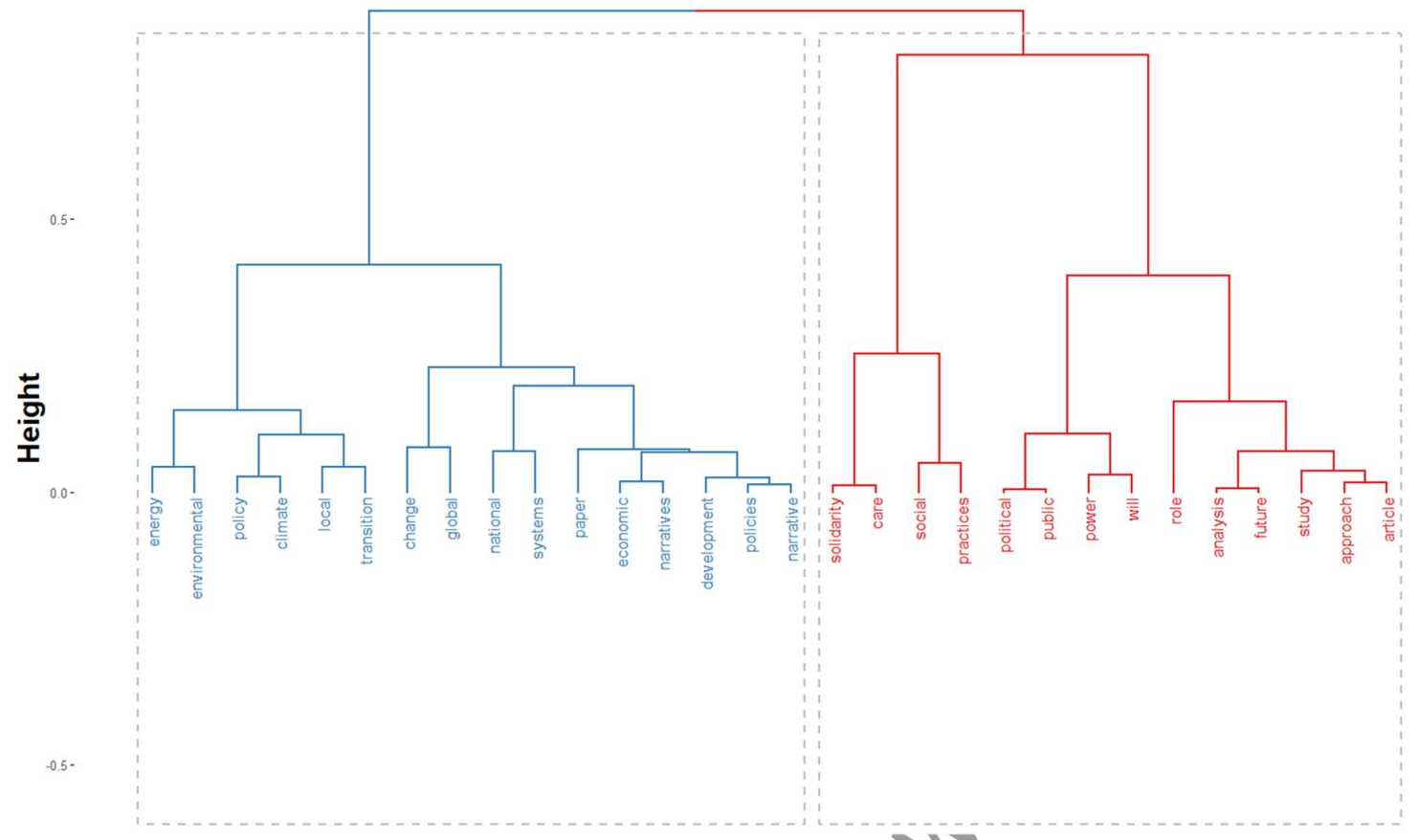

a) Topic dendrogram for keyword set A: energyolicy \& narrative $(n=311)$

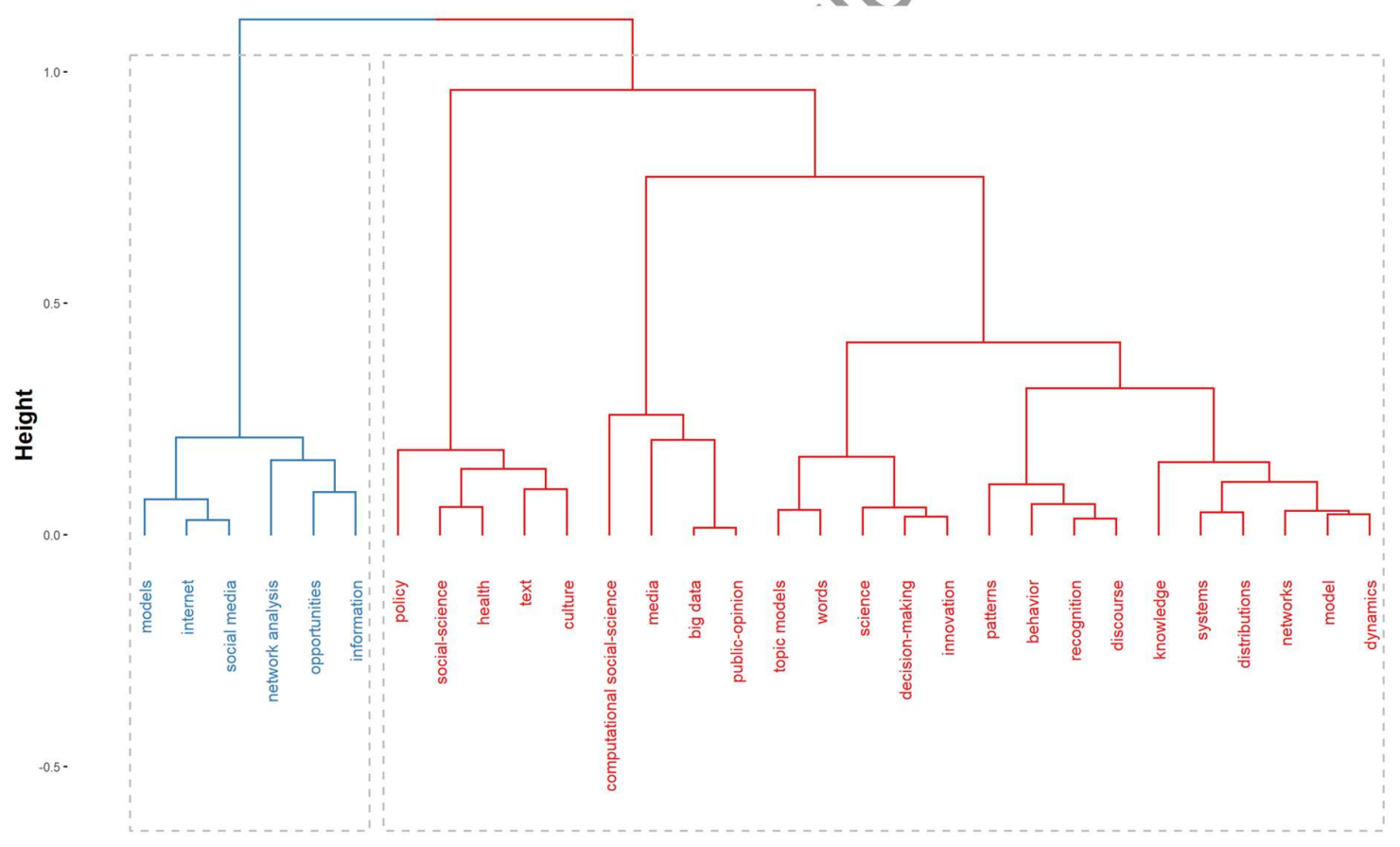

b) Topic dendrogram for keyword set B: computational social science \& topic modelling $(n=94)$

Fig 4. Topic dendrograms demonstrating commonality and cross-fertilisation between A and B as a meta-theoretical fit 
Preprint to be cited as: Debnath, R., Darby, S., Bardhan, R., Mohaddes, K., \& Sunikka-Blank, M. (2020, March 27). A nested computational social science approach for deep-narrative analysis in energy policy research. SocArXiv. March 27. https://doi.org/10.31235/osf.io/hvcb5

\section{Epistemological fit of the proposed methodology}

Topic modelling (TM) is an unsupervised method and inherently inductive approach. It fits well with the scope of grounded theory (GT) and the proposed nested methodology for deep-narrative analysis (see Fig 1). It is opposed to the supervised application of GT for narrative analysis, where the analyst pre-defines categories or scales of the narratives understudy in a deductive fashion. We have argued in section 1 and section 2.1 that such deductive approach induces directionality bias in the interpretation of results. TM purely represents patterns of language use within the text corpus, independent of externalities associated with the text.

It is the analyst's task to interpret and make sense of TM and generate valuable insights based on the semantic relations and crystallised arguments from the narratives. The subjective input of the analyst thus continues to play a vital role and cannot be replaced by TM algorithms for 'good' policy analysis. TM enables the analyst to effectively condense or transform large corpus of narrative texts upon which the analysis realises the analysis itself guided by GT (as per the nested methodology, Fig 1). Fig 5 illustrates the parallel epistemologies that both narrative-analysis and TM represents in the scope of policy analysis. It shows the results from dimensional reduction through factor analysis (see section 4) that clusters similar word/theme from the keyword set A and B from the WoS bibliographic database under study. It represents the epistemological similarity in the themes that are clustered in $A$ and $B$ supporting our claim of epistemological fit of $\mathrm{FM}$ and GT-aided narrative analysis for energy policy research.

Fig 5 indicates the criteria for an epistemological fit of TM and GT-aided nested narrative analysis for energy policy research. It essentially illustrates the type of questions that can be typically investigated using our proposed methodology. For instance, the red clusters in Fig $5 a$ and Fig $5 b$, respectively, thematically hints towards meanings generating characteristic of TM and narrativeanalysis. Words like 'discourse', 'practice', 'behaviour', 'public opinion', 'knowledge', 'distribution' across set $A$ and $B$ represents the application-specific commonality of TM and narrative analysis for energy policy application. It supports the epistemological fit of our proposed methodology in energy policy research and social sciences.

The arguments we developed here concerning the meta-theoretical and epistemological fit between TM and GT-driven narrative analysis centres around the cross-fertilisation and meaning generating capability of both the methods in specific energy policy research context. It is this synergistic capability of both the techniques that strengthen our proposed methodology and do not intend to replace TM or narrative analysis in the contemporary regime of good energy policymaking. The meta-analysis presented here through science mapping of state-of-the-art literature (see Fig 4 and Fig 5) have explained the need for the nested application of computational social science methods (like topic modelling) with established qualitative methods (like grounded theory and narrativeanalysis) to extract the intended insights in policy studies. 
Preprint to be cited as: Debnath, R., Darby, S., Bardhan, R., Mohaddes, K., \& Sunikka-Blank, M. (2020, March 27). A nested computational social science approach for deep-narrative analysis in energy policy research. SocArXiv. March 27. https://doi.org/10.31235/osf.io/hvcb5

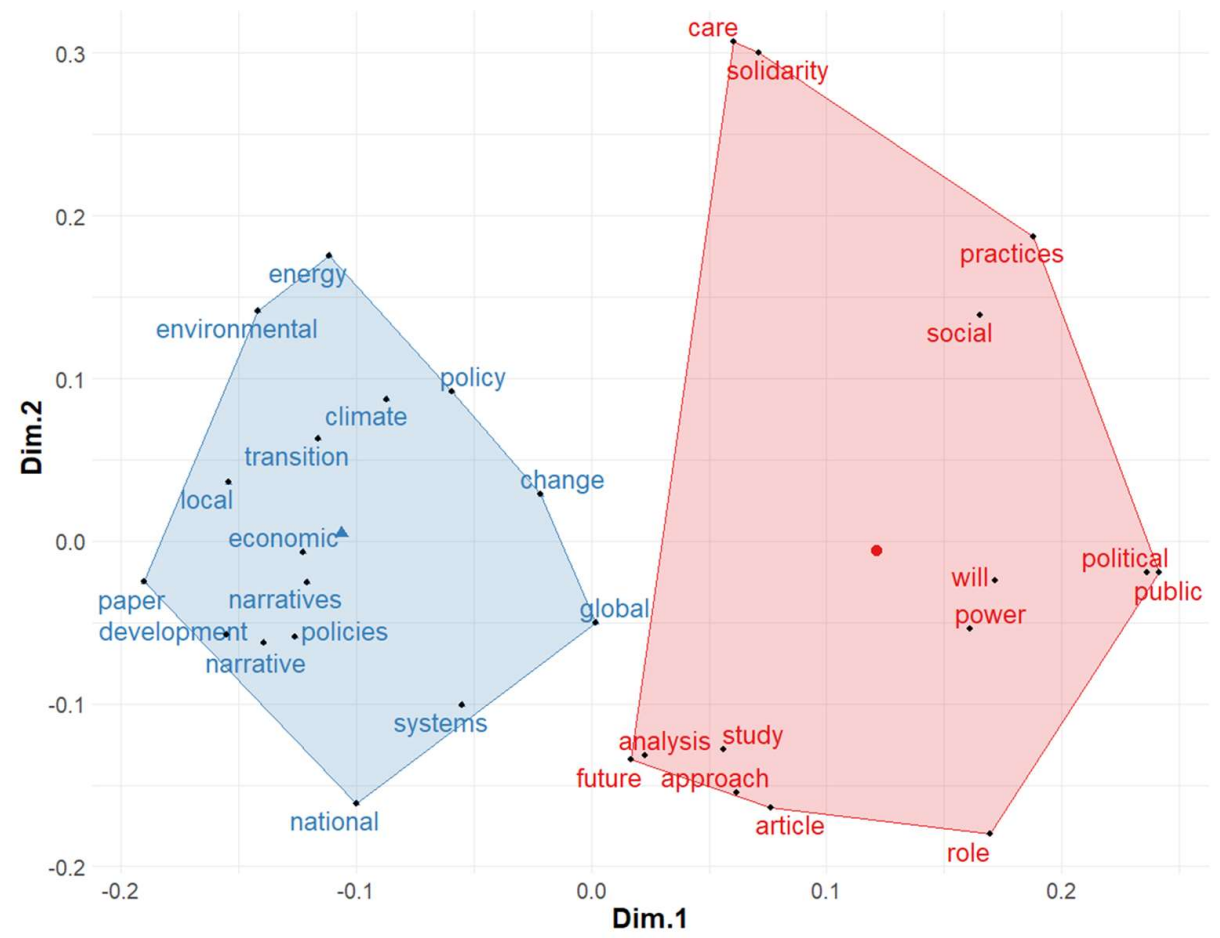

a) Conceptual structure map of themes in keyword set A: energy policy and narrative $(n=311)$

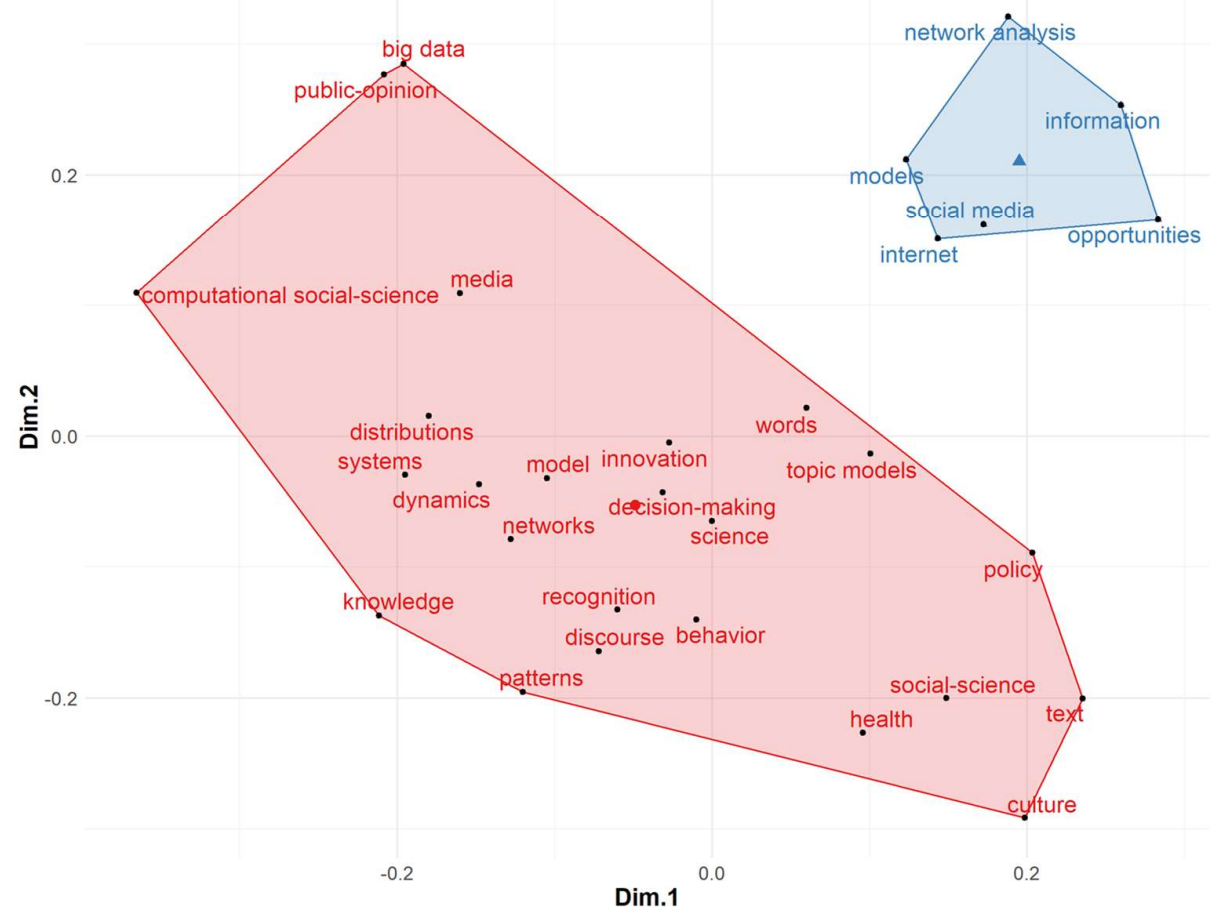

b) Conceptual structure map of themes in keyword set B: computational social science and topic modelling $(n=94)$

Fig 5. Thematic clustering of keyword set A and set B using multidimensional scaling to examine epistemological fit 
Preprint to be cited as: Debnath, R., Darby, S., Bardhan, R., Mohaddes, K., \& Sunikka-Blank, M. (2020, March 27). A nested computational social science approach for deep-narrative analysis in energy policy research. SocArXiv. March 27. https://doi.org/10.31235/osf.io/hvcb5

\section{Concluding remarks}

This study presented a novel nested deep-narrative analysis approach using topic modelling and grounded theory for energy policy application. It emerged from the need of a multidisciplinary approach in energy policy research that transcends beyond the 'technical-only' feasibility of specific scientific methods to address energy and climate injustice (Ozawa et al., 2019). The intention behind the proposed methodology is to contribute to the state-of-the-art literature and strength the use of text-driven policy analysis tools like narratives, stories and storytelling using computational social sciences. Our proposed methodology does not replace qualitative methods like grounded theory or narrative-analysis, rather enhances its insight generating capability by using Bayesian policy modelling and evidence analysis methods based on the laws of probability. We have argued that the combination of probabilistic models with the grounded theory for narrative-analysis may reduce directionality biases in the interpretation of results in energy policy research. The directionality biases are reduced because the proposed model depends solely on the word-word, word-topic and topic-topic interrelationships, defined by probability distribution functions. It enables the analyst/researcher to build the topic models in a way that answers the research questions, rather than adapting research questions so that they can be answered through topic models. It is expected to enhance the insight generation capability of topic modelling in policy research. Walker et al., (2019) argues that the absence of this insight generating capability in the current methods of topic modelling in policy research is a structural limitation.

We argued the potential benefit of combining topic modelling and grounded-theory based narrative analysis, discussed theory theoretical and application-oriented compatibilities using science mapping-based meta-analysis. We also hypothesised a nested methodology that would facilitate their combination and establish the analytical steps. The practical feasibility of the proposed methodology is illustrated in Part-ll of this paper

In the concluding remark, we want to emphasise on the limitation and implications of our methodology. Not all narrative analysis can or should be done using topic modelling techniques as it deconstructs the text corpus into its barebones. The applicability of topic models is up to the judgement of the analyst a case-by-case basis, assessing on the method, the theory and the material at hand. Our proposed method does not solve all the challenges associated with either method, rather it aids in the development of some of the critical questions which systematically includes qualitative evidence into policymaking.

We limit our comment on the applicability of the proposed nested model in a small - dataset, like narratives collected through focussed group discussions or personal interviews. Most applications of topic modelling in the state-of-the-art literature is based on big data, whereas narrative is microdatasets. Sensitive analysis of a big data driven versus a micro-data driven narrative analysis and policy interpretation remains a gap in the literature. While this study did not intend to address this gap, however, we test the practical efficacy of the proposed micro-dataset driven deep-narrative analysis in Debnath et al., (2020). Our meta-analysis based on the bibliographic dataset of the state-of-the-art literature in energy policy, narrative analysis, computational social science and topic modelling have shown that our nested methodology potentially enables in the cross-fertilisation of topic modelling and grounded theory-aided narrative-analysis. It establishes the epistemological and meta-theoretical 
Preprint to be cited as: Debnath, R., Darby, S., Bardhan, R., Mohaddes, K., \& Sunikka-Blank, M. (2020, March 27). A nested computational social science approach for deep-narrative analysis in energy policy research. SocArXiv. March 27. https://doi.org/10.31235/osf.io/hvcb5

fit of our proposed methodology, making it a versatile tool for narrative-based multidisciplinary public policy research.

\section{Acknowledgement}

This study is in part supported by the Bill and Melinda Gates Foundation through the Gates Cambridge Scholarship [OPP1144]. Part of this study is also supported by the Santander Mobility Grant for 2018-19 at the University of Cambridge. All opinion, findings and/or conclusion are that of the authors and do not necessarily reflect the views of the funding organisations.

\section{References}

Aria, M., \& Cuccurullo, C. (2017). bibliometrix: An R-tool for comprehensive science mapping analysis. Journal of Informetrics, 11(4), 959-975. https://doi.org/10.1016/j.joi.2017.08.007

Arun, R., Suresh, V., Veni Madhavan, C. E., \& Murthy Narasimha, M. N. (2010). On Finding the Natural Number of Topics with Latent Dirichlet Allocation: Some Observations. In M. J. Zaki, J. Xu Yu, B. Ravindran, \& V. Pudi (Eds.), Advances in Knowledge Discovery and Data Mining. PAKDD 2010. Lecture Notes in Computer Science (pp. 391-402). Hyderabad: Springer Berlin Heidelberg. https://doi.org/10.1007/978-3-642-13657-3_43

Balasubramanyan, R., Cohen, W. W., Pierce, D., \& Redlawsk, D. P. (2012). Modeling polarizing topics: When do different political communities respond differently to the same news. ICWSM 2012 Proceedings of the 6th International AAAI Conference on Weblogs and Social Media, 18-25.

Bauer, S., Noulas, A., Seaghdha, D. O., Clark, S., \& Mascolo, C. (2012). Talking places: Modelling and analysing linguistic content in foursquare. Proceedings - 2012 ASE/IEEE International Conference on Privacy, Security, Risk and Trust and 2012 ASE/IEEE International Conference on Social Computing, SocialCom/PASSAT 2012, 348-357. https://doi.org/10.1109/SocialComPASSAT.2012.107

Baumer, E. P. S., Mimno, D., Guha, S., Quan, E., \& Gay, G. K. (2017). Comparing grounded theory and topic modeling: Extreme divergence or unlikely convergence? Journal of the Association for Information Science and Technology, 68(6), 1397-1410. https://doi.org/10.1002/asi.23786

Bausch, K. (2008). Negotiating social complexity. In Proceedings of the 52nd Annual Meeting of the ISSS (pp. 1-7). Madison, Wisconsin. Retrieved from http://journals.isss.org/index.php/proceedings52nd/article/download/960/382

Bazeley, P. (2004). Issues in Mixing Qualitative and Quantitative Approaches to Research. In R. Buber, J. Gadner, \& L. Richards (Eds.), 1st International Conference - Qualitative Research in Marketing and Management (pp. 141-156). Vienna: Palgrave Macmilan. Retrieved from https://www.palgrave.com/gp/book/9781403916600

Börner, K., Chen, C., \& Boyack, K. W. (2003). Visualizing knowledge domains. Annual Review of Information Science and Technology, 37, 179-255.

Bryant, A., \& Charmaz, K. (2019). The SAGE Handbook of Current Developments in Grounded Theory. London, UK: SAGE Publications Ltd.

Bushell, S., Buisson, G. S., Workman, M., \& Colley, T. (2017). Strategic narratives in climate change: Towards a unifying narrative to address the action gap on climate change. Energy Research and Social Science, 28(September 2016), 39-49. https://doi.org/10.1016/j.erss.2017.04.001 
Preprint to be cited as: Debnath, R., Darby, S., Bardhan, R., Mohaddes, K., \& Sunikka-Blank, M. (2020, March 27). A nested computational social science approach for deep-narrative analysis in energy policy research. SocArXiv. March 27. https://doi.org/10.31235/osf.io/hvcb5

Callon, M., Courtial, J.-P., Turner, W. A., \& Bauin, S. (1983). From translations to problematic networks: An introduction to co-word analysis. Social Science Information, 22(2), 191-235. https://doi.org/10.1177/053901883022002003

Chen, B., Zhu, L., Kifer, D., \& Lee, D. (2010). What is an opinion about? Exploring political standpoints using opinion scoring model. Proceedings of the National Conference on Artificial Intelligence, 2, 1007-1012.

Cobo, M. J., López-Herrera, A. G., Herrera-Viedma, E., \& Herrera, F. (2011). An approach for detecting, quantifying, and visualizing the evolution of a research field: A practical application to the Fuzzy Sets Theory field. Journal of Informetrics, 5(1), 146-166.

https://doi.org/10.1016/j.joi.2010.10.002

Csardi, G., \& Nepusz, T. (2006). The igraph software package for complex network research. InterJournal, Complex Sy, 1695. Retrieved from https://igraph.org/

De Moya-Anegón, F., Vargas-Quesada, B., Chinchilla-Rodriguez, Z., Corera-Álvarez, E., HerreroSolana, V., \& Munoz-Fernández, F. J. (2005). Domain analysis and information retrieval through the construction of heliocentric maps based on ISI-JCR category cocitation. Information Processing and Management, 41(6), 1520-1533. https://doi.org/10.1016/j.ipm.2005.03.017

Debnath, R., Bardhan, R., Darby, S., Mohaddes, K., Coelho, A. C.V., Isa, A., \& Sunikka-Blank, M. (2020). Energy Justice, Built Environment and Poverty: A Deep-narrative Analysis of Energy Cultures in Brazil, India and Nigeria for Policy Modelling. EngrXiv, March, 1-27. https://doi.org/10.31224/osf.io/8mf46

DiMaggio, P., Nag, M., \& Blei, D. (2013). Exploiting affinities between topic modeling and the sociological perspective on culture: Application to newspaper coverage of U.S. government arts funding. Poetics, 41(6), 570-606. https://doi.org/10.1016/j.poetic.2013.08.004

Fukuyama, H., \& Weber, W. L. (2009). A directional slacks-based measure of technical inefficiency. Socio-Economic Planning Sciences, 43(4), 274-287. https://doi.org/10.1016/j.seps.2008.12.001

Gerlach, M., Peixoto, T. P., \& Altmann, E. G. (2018). A network approach to topic models. Science Advances, 4(7). https://doi.org/10.1126/sciadv.aaq1360

Glaser, B. S., \& Strauss, A. . (1967). The Discovery of Grounded Theory: Strategies for Qualitative Research. New Jersey: Aldine Transactions, A division of transaction publishers.

Griffiths, T. L., \& Steyvers, M. (2004). Finding scientific topics. Proceedings of the National Academy of Sciences of the United States of America, 101(SUPPL. 1), 5228-5235. https://doi.org/10.1073/pnas.0307752101

Grimmer, J., \& Stewart, B. M. (2013). Text as data: The promise and pitfalls of automatic content analysis methods for political texts. Political Analysis, 21(3), 267-297. https://doi.org/10.1093/pan/mps028

Hermwille, L. (2016). The role of narratives in socio-technical transitions - Fukushima and the energy regimes of Japan, Germany, and the United Kingdom. Energy Research and Social Science, 11, 237-246. https://doi.org/10.1016/j.erss.2015.11.001

Howarth, C. (2017). Informing decision making on climate change and low carbon futures: Framing narratives around the United Kingdom's fifth carbon budget. Energy Research and Social Science, 31(October 2016), 295-302. https://doi.org/10.1016/j.erss.2017.06.011

Jacobs, T., \& Tschötschel, R. (2019). Topic models meet discourse analysis: a quantitative tool for a 
Preprint to be cited as: Debnath, R., Darby, S., Bardhan, R., Mohaddes, K., \& Sunikka-Blank, M. (2020, March 27). A nested computational social science approach for deep-narrative analysis in energy policy research. SocArXiv. March 27. https://doi.org/10.31235/osf.io/hvcb5

qualitative approach. International Journal of Social Research Methodology, 22(5), 469-485. https://doi.org/10.1080/13645579.2019.1576317

Janda, K. B., \& Topouzi, M. (2015). Telling tales: using stories to remake energy policy. Building Research and Information, 516-533. https://doi.org/10.1080/09613218.2015.1020217

Jiang, H., Qiang, M., \& Lin, P. (2016). A topic modeling based bibliometric exploration of hydropower research. Renewable and Sustainable Energy Reviews, 57, 226-237. https://doi.org/10.1016/j.rser.2015.12.194

Jockers, M. L. (2013). Macroanalysis: Digital Methods and Literary History (1st ed.). Champaign, IL, USA: University of Illinois Press.

Laouris, Y., Michaelides, M., Damdelen, M., Laouri, R., Beyatli, D., \& Christakis, A. (2009). A systemic evaluation of the state of affairs following the negative outcome of the referendum in cyprus using the structured dialogic design process. Systemic Practice and Action Research, 22(1), 4575. https://doi.org/10.1007/s11213-008-9111-y

Lertzman, R. (2015). Environmental melancholia: psychoanalytic dimensions of engagement. Routledge.

Lui, M., Lau, J. H., \& Baldwin, T. (2014). Automatic Detection and Language Identification of Multilingual Documents. Transactions of the Association for Computational Linguistics, 2, 2740. https://doi.org/10.1162/tacl_a_00163

MacKay, D. J. C. (2008). Sustainable Energy - Without the Hot Air (1st ed.). Cambridge, England: UIT Cambridge Ltd.

Moezzi, M. (2015). Numbers, stories, energy efficiency. In ECEEE Summer Study on Energy Efficiency. European Council for an Energy Efficient Economy.

Moezzi, Mithra, Janda, K. B., \& Rotmann, S. (Eds.). (2017a). Narratives and Storytelling in Energy and Climate Change Research (September). Elsevier. Retrieved from https://www.sciencedirect.com/journal/energy-research-and-social-science/vol/31/suppl/C

Moezzi, Mithra, Janda, K. B., \& Rotmann, S. (2017b). Using stories, narratives, and storytelling in energy and climate change research. Energy Research and Social Science, 31(May), 1-10. https://doi.org/10.1016/j.erss.2017.06.034

Noble, H., \& Mitchell, G. (2016). What is grounded theory? Evidence-Based Nursing, 19(April), 34-35. https://doi.org/10.1136/eb-2016-102306

Ozawa, M., Chaplin, J., Pollitt, M., Reiner, D., \& Warde, P. (Eds.). (2019). In Search of Good Energy Policy. Cambridge, England: Cambridge University Press. https://doi.org/10.1017/9781108639439

Pawson, R. (2002). Evidence-based Policy: The Promise of 'Realist Synthesis'. Evaluation, 8(3), 340358. https://doi.org/10.1177/135638902401462448

Qi, W., Procter, R., Zhang, J., \& Guo, W. (2019). Mapping Consumer Sentiment Toward Wireless Services Using Geospatial Twitter Data. IEEE Access, 7, 113726-113739. https://doi.org/10.1109/access.2019.2935200

Randall, R. (2009). Loss and climate change: the cost of parallel narratives. Ecopsychology, 118-129. https://doi.org/10.1089/eco.2009.0034

Roberts, M. E., Stewart, B. M., \& Tingley, D. (2016). Navigating the Local Modes of Big Data: The Case 
Preprint to be cited as: Debnath, R., Darby, S., Bardhan, R., Mohaddes, K., \& Sunikka-Blank, M. (2020, March 27). A nested computational social science approach for deep-narrative analysis in energy policy research. SocArXiv. March 27. https://doi.org/10.31235/osf.io/hvcb5

of Topic Models. In R. M. Alvarez (Ed.), Computational Social Science (pp. 51-97). Cambridge, England: Cambridge University Press. https://doi.org/10.1017/CBO9781316257340.004

Rotmann, S., Goodchild, B., \& Mourik, R. (2015). Once upon a time... how to tell a good energy efficiency story that sticks. In ECEEE Summer Study on Energy Efficiency. European Council for an Energy-Efficient Economy.

Shaw, C., \& Corner, A. (2017). Using Narrative Workshops to socialise the climate debate: Lessons from two case studies - centre-right audiences and the Scottish public. Energy Research and Social Science, 31(August), 273-283. https://doi.org/10.1016/j.erss.2017.06.029

Sievert, C. (2018). plotly for R. Retrieved from https://plotly-r.com

Skupin, A. (2009). Discrete and continuous conceptualizations of science: Implications for knowledge domain visualization. Journal of Informetrics, 3(3), 233-245. https://doi.org/10.1016/j.joi.2009.03.002

Smith, J., Butler, R., Day, R. J., Goodbody, A. H., Llewellyn, D. H., Rohse, M., ... Whyte, N. M. (2017). Gathering around stories: Interdisciplinary experiments in support of energy system transitions. Energy Research and Social Science, 31(November 2016), 284-294. https://doi.org/10.1016/j.erss.2017.06.026

Song, M., Kim, M. C., \& Jeong, Y. K. (2014). Analyzing the political landscape of 2012 korean presidential election in twitter. IEEE Intelligent Systems, 29(2), 18-26. https://doi.org/10.1109/MIS.2014.20

Strauss, A. L., \& Corbin, J. M. (1997). Grounded theory in practice. SAGE Publications Ltd.

Tang, H., Shen, L., Qi, Y., Chen, Y., Shu, Y., Li, J., \& Clausi, D. A. (2013). A multiscale latent dirichlet allocation model for object-oriented clustering of VHR panchromatic satellite images. IEEE Transactions on Geoscience and Remote Sensing, 51(3), 1680-1692. https://doi.org/10.1109/TGRS.2012.2205579

Törnberg, A., \& Törnberg, P. (2016a). Combining CDA and topic modeling: Analyzing discursive connections between Islamophobia and anti-feminism on an online forum. Discourse and Society, 27(4), 401-422. https://doi.org/10.1177/0957926516634546

Törnberg, A., \& Törnberg, P. (2016b). Muslims in social media discourse: Combining topic modeling and critical discourse analysis. Discourse, Context and Media, 13, 132-142. https://doi.org/10.1016/j.dcm.2016.04.003

Toubia, O., Iyengar, G., Bunnell, R., \& Lemaire, A. (2019). Extracting Features of Entertainment Products: A Guided Latent Dirichlet Allocation Approach Informed by the Psychology of Media Consumption. Journal of Marketing Research, 56(1), 18-36. https://doi.org/10.1177/0022243718820559

Trotter, R. T. (2012). Qualitative research sample design and sample size: Resolving and unresolved issues and inferential imperatives. Preventive Medicine, 55(5), 398-400. https://doi.org/10.1016/j.ypmed.2012.07.003

Vasileiadou, E., Heimeriks, G., \& Petersen, A. C. (2011). Exploring the impact of the IPCC Assessment Reports on science. Environmental Science and Policy, 14(8), 1052-1061. https://doi.org/10.1016/j.envsci.2011.07.002

Vulić, I., De Smet, W., \& Moens, M. F. (2011). Identifying word translations from comparable corpora using latent topic models. ACL-HLT 2011 - Proceedings of the 49th Annual Meeting of the 
Preprint to be cited as: Debnath, R., Darby, S., Bardhan, R., Mohaddes, K., \& Sunikka-Blank, M. (2020, March 27). A nested computational social science approach for deep-narrative analysis in energy policy research. SocArXiv. March 27. https://doi.org/10.31235/osf.io/hvcb5

Association for Computational Linguistics: Human Language Technologies, 2, 479-484.

Walker, R. M., Chandra, Y., Zhang, J., \& van Witteloostuijn, A. (2019). Topic Modeling the ResearchPractice Gap in Public Administration. Public Administration Review, 79(6), 931-937. https://doi.org/10.1111/puar.13095

Wang, Y., \& Taylor, J. E. (2019). DUET: Data-Driven Approach Based on Latent Dirichlet Allocation Topic Modeling. Journal of Computing in Civil Engineering, 33(3). https://doi.org/10.1061/(ASCE)CP.1943-5487.0000819

Williams, M., \& Moser, T. (2019). The Art of Coding and Thematic Exploration in Qualitative Research. International Management Review, 15(1), 45-56. Retrieved from http://scholarspress.us/journals/IMR/pdf/IMR-1-2019/IMR-v15n1art4.pdf

Yano, T., Cohen, W. W., \& Smith, N. A. (2009). Predicting response to political blog posts with topic models. NAACL HLT 2009 - Human Language Technologies: The 2009 Annual Conference of the North American Chapter of the Association for Computational Linguistics, Proceedings of the Conference, (June), 477-485. https://doi.org/10.3115/1620754.1620824

Yao, F., \& Wang, Y. (2019). Tracking urban geo-topics based on dynamic topic model. Computers, Environment and Urban Systems, 79(August 2019), 101419. https://doi.org/10.1016/j.compenvurbsys.2019.101419

Zirn, C., \& Stuckenschmidt, H. (2014). Multidimensional topic analysis in political texts. Data and Knowledge Engineering, 90, 38-53. https://doi.org/10.1016/j.datak.2013.07.003 